International Journal of Pure and Applied Mathematics

Volume $96 \quad$ No. $3 \quad 2014,323-327$

ISSN: 1311-8080 (printed version); ISSN: 1314-3395 (on-line version)

url: http://www.ijpam.eu

doi: http://dx.doi.org/10.12732/ijpam.v96i3.3

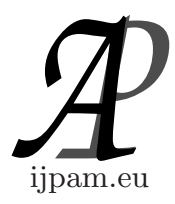

\title{
REFINEMENTS OF RAMANUJAN FORMULA FOR GAMMA FUNCTION
}

\author{
Sorinel Dumitrescu ${ }^{1}$, Cristinel Mortici ${ }^{2} \S$ \\ ${ }^{1}$ University Politehnica of Bucharest \\ Splaiul Independen ței 313, Bucharest, ROMANIA \\ ${ }^{2}$ Valahia University of Târgovişte
}

Bd. Unirii 18, 130082 Târgovişte and Academy of Romanian Scientists Splaiul Independenţei 54, 0500594 Bucharest, ROMANIA

AMS Subject Classification: 33B15, 26D15, 41A25, 34E05

Key Words: gamma function, Ramanujan formula

\section{Introduction}

Many researchers have paid great attention to the problem of approximating the gamma function. Undeniebly the most known formula is Stirling's formula, e.g., [1],

$$
\Gamma(x+1) \sim \sqrt{2 \pi x}\left(\frac{x}{e}\right)^{x} .
$$

Slightly better results give Burnside formula [3]

$$
\Gamma(x+1) \sim \sqrt{2 \pi}\left(\frac{x+\frac{1}{2}}{e}\right)^{x+\frac{1}{2}}
$$

and Gosper's formula [4]

Received: May 5, 2014

(c) 2014 Academic Publications, Ltd.

$\S$ Correspondence author url: www.acadpubl.eu 


$$
\Gamma(x+1) \sim \sqrt{2 \pi x}\left(\frac{x}{e}\right)^{x} \sqrt{1+\frac{1}{6 x}} .
$$

However Ramanujan formula, e.g., [2], [5]-[11]

$$
\Gamma(x+1) \sim \rho(x):=\sqrt{2 \pi x}\left(\frac{x}{e}\right)^{x} \sqrt[6]{1+\frac{1}{2 x}+\frac{1}{8 x^{2}}+\frac{1}{240 x^{3}}}
$$

is more accurate. We propose in this note the replacing the terms in powers in $x^{-k}$ by powers in $(x-m)^{-k}$, where $m$ is certain real number. More precisely, we introduce the following class of approximations for every $m, b, c \in \mathbb{R}$ :

$$
\Gamma(x+1) \sim \sqrt{2 \pi x}\left(\frac{x}{e}\right) \sqrt[x]{1+\frac{1}{2(x-m)}+\frac{b}{(x-m)^{2}}+\frac{c}{(x-m)^{3}}} .
$$

Note that Ramanujan's formula is obtained in $m=0, b=\frac{1}{8}, c=\frac{1}{240}$ case. We show that there are other values of $m, b, c$ for which better approximations can be obtained.

\section{The Results}

Some individual computations we made motivated us to introduce the following class of approximations for every $a \in \mathbb{R}$ :

$$
\begin{aligned}
& \Gamma(x+1) \sim \\
& \quad \sqrt{2 \pi x}\left(\frac{x}{e}\right) \sqrt[x]{1+\frac{1}{2\left(x+2 a-\frac{1}{4}\right)}+\frac{a}{\left(x+2 a-\frac{1}{4}\right)^{2}}+\frac{2 a^{2}-\frac{13}{480}}{\left(x+2 a-\frac{1}{4}\right)^{3}}},
\end{aligned}
$$

which is a sub-family of (2).

Lemma 1. Let $a>\frac{1}{8}$. If (3) is an upper-approximation, then it is more accurate than Ramanujan's formula.

Proof. Known fact, Ramanujan's formula (1) is an upper-approximation of gamma function. Our assertion follows if we prove that

$$
\sqrt[6]{1+\frac{1}{2\left(x+2 a-\frac{1}{4}\right)}+\frac{a}{\left(x+2 a-\frac{1}{4}\right)^{2}}+\frac{2 a^{2}-\frac{13}{480}}{\left(x+2 a-\frac{1}{4}\right)^{3}}}
$$




$$
<\sqrt[6]{1+\frac{1}{2 x}+\frac{1}{8 x^{2}}+\frac{1}{240 x^{3}}} .
$$

In this sense, we have

$$
\begin{aligned}
& \left(1+\frac{1}{2\left(x+2 a-\frac{1}{4}\right)}+\frac{a}{\left(x+2 a-\frac{1}{4}\right)^{2}}+\frac{2 a^{2}-\frac{13}{480}}{\left(x+2 a-\frac{1}{4}\right)^{3}}\right) \\
& -\left(1+\frac{1}{2 x}+\frac{1}{8 x^{2}}+\frac{1}{240 x^{3}}\right) \\
= & -\frac{(8 a-1) T(x)}{240 x^{3}(4 x+8 a-1)^{3}} \\
< & 0,
\end{aligned}
$$

where

$T(x)=\left(7680 a^{2}+960 a-192\right) x^{2}+\left(1920 a^{2}-384 a+18\right) x+\left(64 a^{2}-16 a+1\right)$.

As $a>\frac{1}{8}$, all coefficients of the polynomial $T$ are positive, so $T>0$. The proof is now completed.

For a concrete comparison, let us take $a=\frac{1}{7}$ in (3) to get

$$
\begin{aligned}
\frac{\Gamma(x+1)}{\sqrt{2 \pi x}\left(\frac{x}{e}\right)^{x}} \sim \beta(x) & \\
: & =\sqrt[6]{1+\frac{1}{2\left(x+\frac{1}{28}\right)}+\frac{1}{7\left(x+\frac{1}{28}\right)^{2}}+\frac{323}{23520\left(x+\frac{1}{28}\right)^{3}}} .
\end{aligned}
$$

The next table shows us the superiority of $\Gamma(x+1) \sim \beta(x)$ over Ramanujan's formula $\Gamma(x+1) \sim \rho(x)$.

\begin{tabular}{|l|l|l|}
\hline$x$ & $\ln \{\rho(x) / \Gamma(x+1)\}$ & $\ln \{\beta(x) / \Gamma(x+1)\}$ \\
\hline 10 & $8.5872 \times 10^{-8}$ & $7.0976 \times 10^{-8}$ \\
\hline 25 & $2.3455 \times 10^{-9}$ & $1.9506 \times 10^{-9}$ \\
\hline 100 & $9.4519 \times 10^{-12}$ & $7.8819 \times 10^{-12}$ \\
\hline 500 & $1.5247 \times 10^{-14}$ & $1.2723 \times 10^{-14}$ \\
\hline
\end{tabular}

By replacing 323 by 321 in (4), an under-approximation is obtained,

$$
\frac{\Gamma(x+1)}{\sqrt{2 \pi x}\left(\frac{x}{e}\right)^{x}} \sim \alpha(x):=\sqrt[6]{1+\frac{1}{2\left(x+\frac{1}{28}\right)}+\frac{1}{7\left(x+\frac{1}{28}\right)^{2}}+\frac{321}{23520\left(x+\frac{1}{28}\right)^{3}}},
$$


as we can see from the following theorem.

Theorem 1. The following inequalities are valid for every $x \geq 75$ in the left-hand side and for every $x \geq 2$ in the right-hand side:

$$
\alpha(x)<\frac{\Gamma(x+1)}{\sqrt{2 \pi x}\left(\frac{x}{e}\right)^{x}}<\beta(x) .
$$

Proof. We use the following inequalities:

$$
\exp \{u(x)\}<\frac{\Gamma(x+1)}{\sqrt{2 \pi x}\left(\frac{x}{e}\right)^{x}}<\exp \{v(x)\},
$$

where

$$
\begin{gathered}
u(x)=\frac{1}{12 x}-\frac{1}{360 x^{3}}+\frac{1}{1260 x^{5}}-\frac{1}{1680 x^{7}}, \\
v(x)=\frac{1}{12 x}-\frac{1}{360 x^{3}}+\frac{1}{1260 x^{5}}-\frac{1}{1680 x^{7}}+\frac{1}{1188 x^{9}} .
\end{gathered}
$$

It remains to show that $f<0$ and $g<0$, where

$$
f(x)=-u(x)+\ln \alpha(x), \quad g(x)=v(x)-\ln \beta(x) .
$$

This is true, since $f, g$ are strictly increasing on $[75, \infty)$ and $[2, \infty)$ respectively, with $f(\infty)=g(\infty)=0$. For the monotonicity,

$$
\begin{gathered}
f^{\prime}(x)=\frac{P(x)}{5040 x^{8}(28 x+1)\left(20020 x+66640 x^{2}+109760 x^{3}+2133\right)}, \\
g^{\prime}(x)=\frac{Q(x)}{55440 x^{10}(28 x+1)\left(60060 x+199920 x^{2}+329280 x^{3}+6427\right)},
\end{gathered}
$$

where $P$ and $Q$ are polynomials with positive leading coefficient having the largest real roots $74.398 \cdots$ and $1.372 \cdots$, respectively.

Finally we pose the open problem of finding the values of $m, a, b, c$ which provide the most accurate approximations (2) and (3).

\section{Acknowledgments}

The work of the second author was supported by a grant of the Romanian National Authority for Scientific Research, CNCS-UEFISCDI project number PN-II-ID-PCE-2011-3-0087. 


\section{References}

[1] M. Abramowitz and I. A. Stegun, eds. Handbook of Mathematical Functions with Formulas, Graphs, and Mathematical Tables, 9th printing, In : National Bureau of Standards, Applied Mathematical Series, 55 (1972), Dover, New York.

[2] B.C. Berndt, Y.-S. Choi, S.-Y. Kang, The Problems Submitted by Ramanujan, J. Indian Math. Soc., Contemporary Math. 236 (1999), 15-56.

[3] W. Burnside, A rapidly convergent series for $\log N$ !, Messenger Math., 46 (1917), 157-159.

[4] R.W. Gosper, Decision procedure for indefinite hypergeometric summation, Proc. Natl. Acad. Sci. USA, 75 (1918), 40-42.

[5] C. Mortici, Ramanujan formula for the generalized Stirling approximation, Appl. Math. Comp., 217 (2010), 2579-2585.

[6] C. Mortici, C.-P. Chen, On the harmonic number expansion by Ramanujan, J. Inequal. Appl., 2013 (2013), art. no. 222.

[7] C. Mortici, An improvement of the Ramanujan formula for approximation of the Euler Gamma function, Carpathian J. Math., 28 (2012), 285-287.

[8] C. Mortici, Error estimates of Ramanujan-type series, Ramanujan J., 27 (2012), 169-179.

[9] C. Mortici, On Ramanujan's large argument formula for the gamma function, Ramanujan J., 26 (2011), 185-192.

[10] C. Mortici, Ramanujan's estimate for the gamma function via monotonicity arguments, Ramanujan J., 25 (2011), 149-154.

[11] S. Ramanujan, The lost notebook and other unpublished papers, Intr. by G. E. Andrews, Narosa Publ. H.-Springer, New Delhi-Berlin, 1988. 
